\title{
Method to mix tea with honey and use of transient light attenuation as an indicator of homogeneity
}

\author{
Henrique Ferreira, Ricardo R. Gitti, Andre J. Ferreira-Martins, Matheus Pessoa*, Pedro R. Sardelich, and \\ Isabela S. Bijotti \\ Universidade Federal do ABC - 5001, Avenida dos Estados, Santo Andre, Sao Paulo, Brazil
}

Received: 30 September 2017 / Accepted: 25 April 2018

\begin{abstract}
We built a magnetic stirrer to mix tea and honey and developed a method to determine the homogeneity of the mixture based on the variation of light intensity of a laser beam with time due to its scattering by the mixture. The addressed problem was that of mixing tea and honey as fast as possible with a power limitation of one single $1.5 \mathrm{~V}$ battery, following the statement of Problem 15 of the 9th International Physicists' Tournament. The influence of temperature and honey concentration on the total time of mixing was studied through the performance of several experiments reported here. In the best configuration of parameters, the total mixture occurs in less than $2 \mathrm{~s}$, while in the worst configuration, the time necessary reaches $10 \mathrm{~s}$. Results validate the magnetic stirrer as a proper device for mixing tea with honey and light attenuation as an excellent physical property for investigating the homogeneity of the mixture of the honey in tea. Supplementary investigations were made exploring the influence of adding sugar and or a slice of lemon to the tea.
\end{abstract}

Keywords: Light scattering / magnetic stirrer

\section{Introduction}

Tea is undoubtedly among the most consumed drinks in the world. Although variations may occur due to personal taste, tea is often consumed with a sweetener. In this paper, we will consider a common choice: tea sweetened with honey.

Direct experience shows that mixing tea with honey may sometimes not be the easiest task: given the fluids' low apparent diffusivity [1], it is often necessary to employ considerable effort into the stirring process for the complete mixture to occur. Therefore, it may be useful to develop a stirrer device, which automates such task. From that, two questions naturally arise: first, what is the optimum design of such device? And secondly, how can one be sure when the honey is totally mixed in tea? These are the main questions addressed in this paper.

The first question is too broad: in order to determine an optimal design, it is first necessary to establish the limitations under which the device is subjected, as well as the parameters which are supposed to be optimized. It was considered as a limitation the power source of the device, which will be constrained to be operated with a single $1.5 \mathrm{~V}$ battery. The optimization parameter, on the other hand, is clear: build a device capable of uniformly mixing honey in tea as fast as possible.

\footnotetext{
* e-mail: matheusp97@hotmail.com
}

In what concerns the second question, the condition to be imposed is that the homogeneity determination method should not directly interfere in the mixture. Considering this, it is necessary to develop a method based on exterior interferences only, which directly excludes the option of adding any substance to the tea apart from honey.

With these conditions established, the problem addressed in this paper is fully specified, as it was originally proposed in the 9th International Physicists' Tournament. ${ }^{1}$

It was found out that the optimal stirrer design is a magnetic stirrer, and the developed homogeneity criteria method was based on transient light attenuation. These choices will be promptly presented and discussed further on this introductory section. In Section 2, a detailed discussion about the device and experimental setup is presented. Even though most of the paper focuses on the physical discussion and experimental considerations of the magnetic stirrer and light scattering process, several attempts were made in order to determine the stirrer's optimum design and the best homogeneity criteria method. These attempts will be briefly discussed in Section 5 . We will then be able

\footnotetext{
1 In this paper we report the solution of for Problem 15 of the 9th International Physicists' Tournament, as can be found in: http:// 2017.iptnet.info/problems/. The solution here presented was developed by the Brazilian Team, consisting of this paper's authors, which took part in the Tournament.
} 


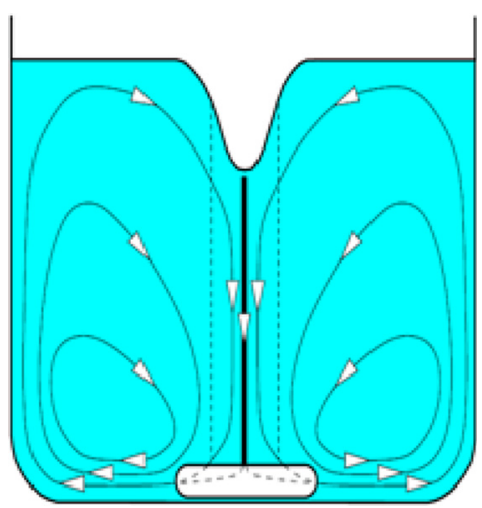

Fig. 1. Fluid flow under vortex dynamics. Continuous lines are streamlines, the bold line is the downward jet, and the dashed line represent the average location of dye curtains, from reference [2].

to conclude the present study, in Section 6, taking into consideration the many attempts which led to the development of the best method to address the aforementioned problem.

To start our physical considerations and introduce the motivation for our investigation, we will discuss the physical processes directly related to our solution to the addressed problem. First, we discuss the mixing process of tea and honey, by making considerations on the motion of the fluid inside a vessel once it is stirred, and why this aspect is important when studying the problem of mixing tea with honey. After this, we will present the chosen design for the sought device: a magnetic stirrer, whose mechanics is shortly after discussed. We then turn our attention to the description and discussion of the chosen homogeneity determination method: the measurement of the difference in light intensity with time due to its attenuation by particulates in the mixture.

\subsection{Transport mechanisms}

Transport mechanisms in fluids may be determined by different processes such as diffusion or drift. Fick's laws describe the behavior of diffusing molecules as a consequence of concentration gradients: the first law in one dimension is an expression for the flow of particles from high to low concentration, which takes into account the velocity with which diffusion will occur on the system; whereas the second law states how diffusion will influence concentration in a time-dependent relation.

In stationary states without external interference, the diffusion process occurring accordingly to the concentration gradient is slow, driven by the natural thermal motion. Such natural process is insufficient to provide a time-optimized mixing of honey and tea, as the problem to which we are addressing states. Therefore, in order to increase the mixing rate and mix tea with honey rapidly, work has to be done by an external agent which stirs the mixture.

The aim of the stirring process is to create a strong fluid motion in the form of a vortex (as Fig. 1), believed to be the reason for the method's efficient stirring due to the induced fluid convection, as detailed in [2]. In our case, the stirring agent which will provide the necessary

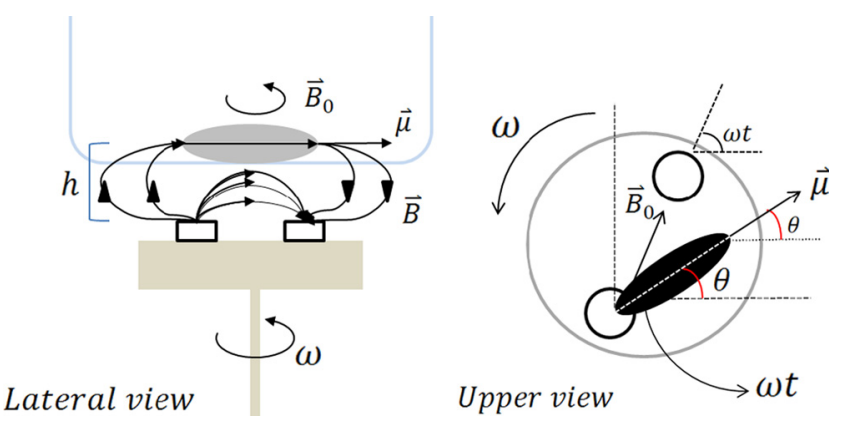

Fig. 2. Schematic of the magnetic bar movement due to magnetic torque transfer from the rotating magnet. Lateral and top views.

external work will be the magnetic stirrer - while, of course, the provided mechanical work essentially comes from the $1.5 \mathrm{~V}$ battery which powers the stirrer.

\subsection{Magnetic stirrer}

The magnetic stirrer is a common laboratory tool used to mix substances by creating a vortex within a vessel. The device consists in two permanent neodymium magnets glued (with opposite poles facing up) to a plastic gear, centered to the axis of a DC clockwise motor which is the powered by the battery. The DC motor is, therefore, the responsible for the rotation of the gear and magnets, thus, it is fundamentally the source of the stirring.

The stirring process happens as the magnets placed below the vessel induce a magnetic torque to a magnetized bar placed within the vessel - as shown in the lateral view of Figure 2 -, which then makes the bar to rotate following the magnets bellow it. The rotating stirrer bar drags an amount of fluid within the vessel which creates a vortex; it is fast, portable, silent and energetically efficient since the high coupling between the magnet and the magnetic bar decreases mechanical energy losses, which is also supported by the low heat and sound dissipation. The stirring bar is covered by Teflon, a material that does not easily stick with honey, preventing any residual non-dissolved honey from lowering the efficiency of the method.

The limitation establishing that the magnetic stirrer should be powered by a single $1.5 \mathrm{~V}$ AA battery was one of the most important ones when choosing the stirrers design. As it will be discussed in Section 5, purely mechanical stirrers could not be powered by such a limited source. After many attempts, we found out that it is perfectly possible to build an efficient magnetic stirrer powered only by an AA battery, but no other design was possible since other stirrers would require a greater operational tension.

\subsection{Homogeneity criteria}

The behavior of the laser light passing through the tea is described by the theory of the interaction between light and matter.

When a light beam passes through a particulate medium it is expected an attenuation in light intensity. Such phenomenon, known as extinction, happens due to light absorption and scattering processes (as Fig. 3), though one of the processes may dominate over the 


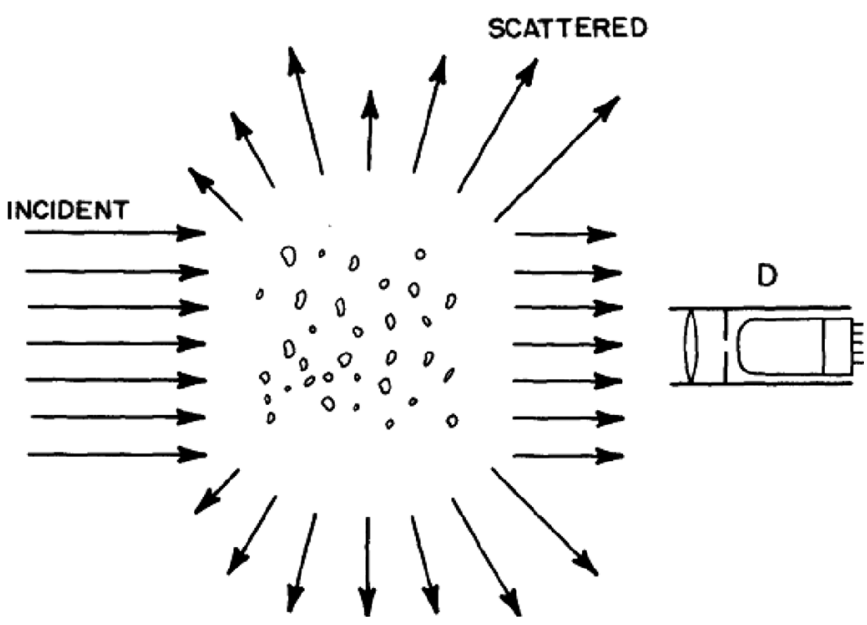

Fig. 3. Illustration of the light being scattered by particulates, which decreases its intensity as measured by a detector [5].

other, depending mostly on the size and density of the particles of the medium. Honey is approximately $69.5 \%$ [3] constituted of glucose and fructose arranged in molecular complexes, which molecular diameters make scattering phenomena dominate over absorption [4].

Light attenuation through a medium is described by the Lambert-Beer law, which relates transmittance of a medium where radiation is propagated with the probability of interaction of radiation and particles in this medium [6]. This probability is described by these particles cross section and by their concentration [7]. The relation between the incident, $I_{0}$, and attenuated, $I_{a}$, light intensity which travels a distance $d$ in the medium with particle density $N$. The light intensity decays exponentially with the cross section $\sigma$, according to equation (1):

$$
I_{a}=I_{0} e^{-\sigma N d}
$$

Light attenuation happens due to different physical phenomena of interaction between light and matter. Light extinction can occur because of light scattering or absorption by the molecules. Light scattering changes the reflection angles of light, which causes a diffuse reflection (in case of an elastic scattering). The extinction cross section is determined by the contribution of two cross sections: scattering and absorption ones [7]. The predominance of each phenomenon depends on the size of the particles interacting with light. Therefore inelastic scattering may also occur, like Raman scattering $[8,9]$, which the scattered light gives vibrational energy to the interacted molecules.

We acknowledge the existence of two different elastic scattering phenomena that can be related to our problem: Mie's and Rayleigh's. The differences between Mie's scattering and Rayleigh's scattering phenomena consist of the ratio between the particles and the incident light wavelength. If the ratio of particles diameter divided by the wavelength is up to 0.1 , it is considered a Rayleigh scattering, and the particles scatter the incident wavelengths with different angles, which divides the spectrum [10]. Mie's ratio is closer or higher than 1 [11]. In practice, the equations of light scattering are the same for both, although the distinction between the processes is qualitatively important.

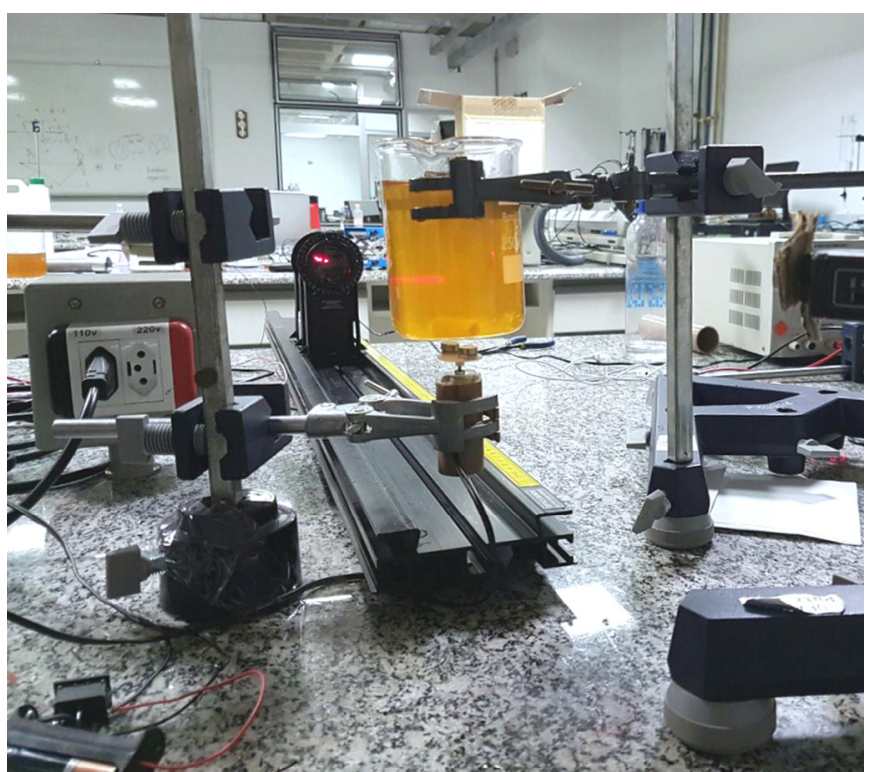

Fig. 4. Magnetic stirrer built to mix tea and honey. At the back, the laser used to measure light scattering.

The honey used is mostly composed of glucose, fructose and sucrose. This last one is a disaccharide that has a molecular diameter of $0.86 \mathrm{~nm}$ [12]. We used a single laser with a specific wavelength of $635 \mathrm{~nm}$, which means that the ratio between the particles and the light wavelength is approximately 0.0014. Thus, the scattering happens according to Rayleigh's theory. Although the Rayleigh scattering is the correct process describing the phenomenon, the scattering in different angles according to the wavelength does not occur, considering that we use a laser, which is monochromatic.

If honey is dissolved creating small molecular aggregates (in our case, no more than approximately 70 molecules per aggregate), Rayleigh scattering is the major effect. If there are presence of crystals and bigger molecular aggregates, Mie scattering effect will be more relevant.

The difference of transmittance of the medium before and after the mixing can be used as an homogeneity criteria, once it relates the phenomenon with the concentration of molecules of a substance through the analyzed path. While transmittance is not constant, it means that concentrations of the substances are changing. Once transmittance keeps constant, it indicates that the medium is homogeneous.

\section{Method}

In this section, we describe in detail the experimental setup and method used to determine the dissolution time of honey in tea. The developed methodology aims to provide results describing how well (i.e., uniformly) honey and tea are mixed over time. Different experiments were performed under varied conditions, as it will be specified.

Mechanical supports are used to place the motor beneath a beaker, the vessel in which the tea and the honey is placed. The magnet bar is then placed at the bottom of the beaker, as shown in Figure 4. 


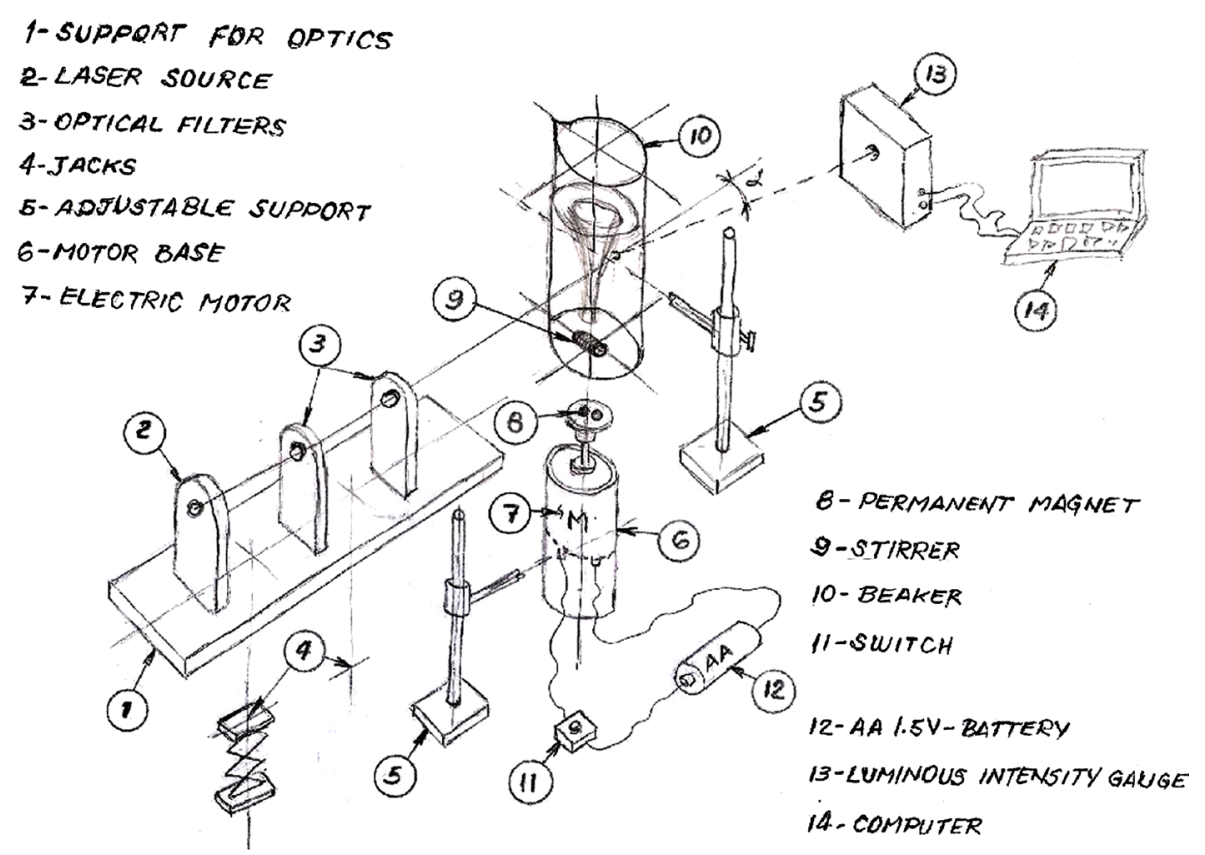

Fig. 5. Representation of the employed experimental setup.

A red diode laser (635 nm, $0.9 \mathrm{~mW}$, Pasco OS-8525A) is used as light source. The laser is directed through two consecutive linear polarization filters, which is used to control the incident laser light intensity by controlling the orientation of one of the linear polarizer with respect to the other, as described by Malus' law, which relates the intensity of the light that passes through a polarizer with the angle between the light's initial direction and the axis of the polarizer [13].

The laser beam crosses the beaker off-axis such that it will not cross the vortex, crossing only the glass and tea, resulting in a refraction. The best position of the laser across the vessel was chosen by the following criteria: it must be a region which light is not disturbed by the vortex formation or by the magnetic bar. With this, we tested different heights of the laser in relation to the vessel's bottom, trying to see if the results of homogeneity could be different. We discovered that the position of the laser does not influence the results once it does not pass through the vortex.

A photodetector was placed after the beaker in a path that intercepts the incoming refracted beam, allowing the attenuated light intensity to be acquired by a computer via a USB interface. Two jacks are positioned below the laser source and filters are used to regulate laser's intensity. For more precise results, the experiment took place in a dark room.

With the beaker filled with tea, the lasers intensity level is then regulated adjusting the polarizer to $80 \%$ of the detector's full scale. Intensity does not change considerably if water is static or under vortex flow (if the laser does not cross the vortex). The full experimental setup can be seen in Figure 5.

A typical experimental trace is presented in Figure 6 . The recording is started before honey is added to the tea - this is characterized by the $80 \%$ intensity level plateau in region 1 . Afterward, honey is added to the tea, which causes an abrupt decrease in the light intensity, which happens as the honey chunks move to the bottom, which causes a transient light scattering, as seen in region 2. After some time, the light intensity is again stabilized, but in a lower plateau, seen as region 3. This happens because some of the honey already diffused through the tea, making the medium more opaque. When the DC motor is started, the stirring creates a vortex which vigorously moves the honey molecules all around the beaker and the intensity falls sharply, dropping to zero intensity near $t=50 \mathrm{~s}$. In region 4 , we notice more data irregularity, and it happens because air bubbles are passing through the laser when the stirrer is working, but on average, the curve stabilizes in a new and final plateau. The decay noticed after the quick homogeneity in $t=50 \mathrm{~s}$ is due to variation of thermal energy of the system.

This final intensity level corresponds to the final mixed configuration where the mixed fluid will have a different viscosity, color, density of particles, etc. The constancy of this intensity occurs because the light is being scattered by evenly distributed particles, which attests the homogeneity of the dispersion. All the honey/sugar is completely diluted as confirmed by visual inspection, no honey/sugar is at the bottom of the beaker.

Notice in Figure 6, at the end of region 2, that natural diffusion makes no significant contribution to mixing the fluids, once the intensity level has not suffered significant reduction as compared to the final configuration of region 4 . With those data collected, the region 3 figures are fitted by an exponential curve in which the exact time when it stabilizes can be noticed. The fitting function is given by equation (2):

$$
y=y_{0}+A e^{-x / \tau} .
$$

Equation (2) describes the transient process of honey dispersion caused by the vortex. The constant time $\tau$ represents the time response of the system, and after five $\tau$ the function reaches a value superior to 0.99 of its final value. 


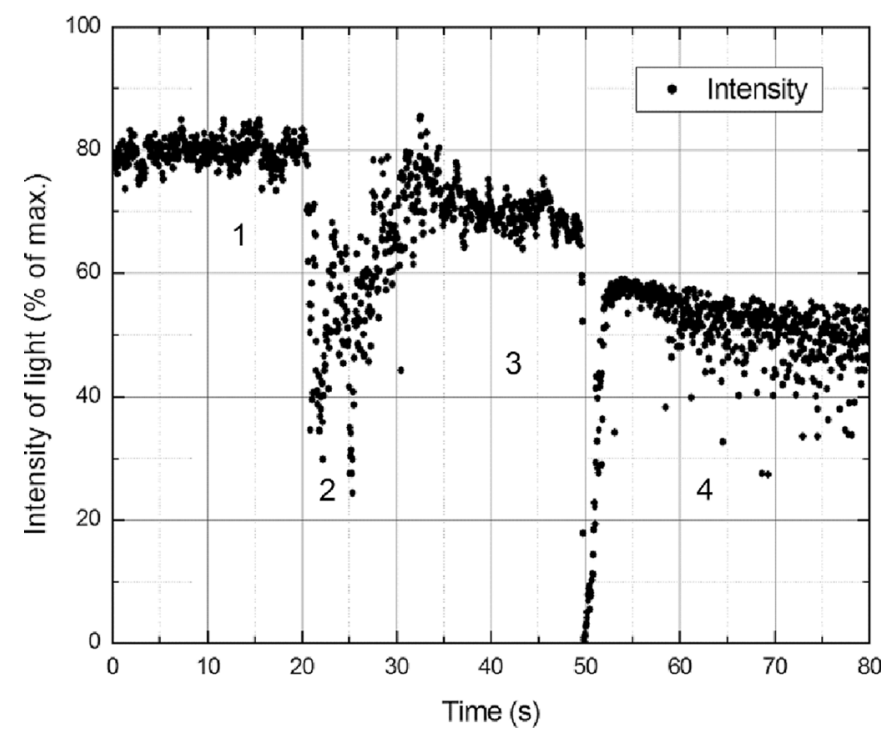

Fig. 6. Typical experimental time trace. Region 1: the stirrer is off, corresponding to an average intensity of $80 \%$. Region 2: At $t=20 \mathrm{~s}$, honey is poured and some fluctuations are recorded, but most honey falls to the bottom. At this point, there is also an influence of diffusion process, noticed at $t=35 \mathrm{~s}$. Region 3 : the stirrer is turned on causing the sudden drop in light intensity. Region 4: the pattern of an exponential decay is noticed in a short period of time at $t=50 \mathrm{~s}$, providing information about the fluid's homogeneity through light intensity measurements. In $t=80 \mathrm{~s}$, the stirrer is turned off and there is no longer data acquisition.

\section{Results}

Here, we present results for different parts we investigated. The first one about mechanical and electrical conditions, since the magnetic stirrer is one of many options one can mix tea with honey (besides a spoon and other methods); we were able to investigate some variables such as the direction to which the magnet is being rotated and the influence in its velocity and also investigated quantitatively the influence of the magnetic coupling between the magnetic bar and the magnet at the top of the motor. The conclusion about the motor's RPM rate being larger without any coupling can be inferred easily when performing experiments but quantitative measurements provide more accuracy to it. The main experiments that are deeply discussed are the influence of concentration and temperature on the results for final solubilization of tea with honey.

\subsection{Optimal mechanical and electrical conditions}

The system was improved in order to transmit the most torque as possible to the fluid. The magnetic bar is attracted by the magnetic field, which intensifies its contact force with bottom glass of the beaker. If the magnets and the bar are too close, the friction between bar and cup is strong enough to impede that the bar reaches the rotation of the gear. On the other hand, if bar and magnets are too far, the total torque transmitted weakens, see equation (8), until the magnets make no considerable effect to the bar. The bar itself was not varied in our experimental approximations.

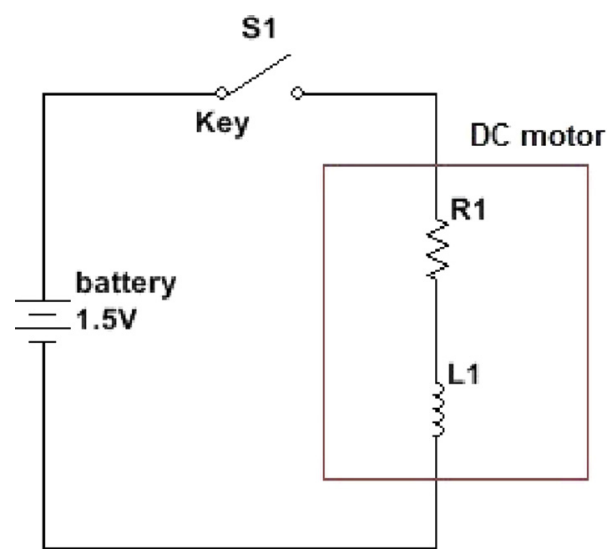

Fig. 7. Electric circuit of the stirrer apparatus.

Considering this, there is an optimal distance between the bar and magnets able to transmit more torque to the system. Experimentally, with a precision of $0.5 \mathrm{~mm}$, we found that a $9.0 \mathrm{~mm}$ distance has the optimal efficiency. Using a tachometer, the quotient between motors RPM with and without the influence of the magnetic bar was determined. Once we used a clockwise motor, we expected and confirmed that the best efficiency is reached in this sense. The RPM is important since whenever we add sugar or honey to the system, the resulting viscous drag influences the movement according to equation (10).

Table 1 shows the difference in efficiency when using a motor in its clockwise natural position and in counterclockwise sense; RPM measures were made with the help of a tachometer.

Since a clockwise turn has a slightly higher RPM value and efficiency, we choose this direction for executing all experiments. We also found that current and tension provided by the 1.5 AA battery changes when the magnetic bar is attracted by the magnetic field. Tension decreases and current increases, as we show in Table 2.

This happens because when the bar is coupled to it, the gear provides more current to rotate. Thus the more power is needed due to the electromagnetic attraction. The total power of the system was calculated by multiplying the stirrer's current and tension $(1.8 \pm 0.1) \mathrm{W}$. Part of this power goes only for gear's rotation $(1.1 \pm 0.1) \mathrm{W}$. In this setup, $0.7 \mathrm{~W}$ is being transferred to the magnetic bar.

The circuit setup used in our experiments it consists of a battery, a switcher S1 and the DC motor (with internal electric resistance R1 and electric inductance L1) in a series circuit (Fig. 7).

\subsection{Influence of concentration}

Experimentally, it was noticed that increasing the amount of honey increases the time needed for homogeneity, as expected. These tests were made at room temperatures of approximately $30.0^{\circ} \mathrm{C}$. We can see that lower concentration values of honey are mixed faster. Although, among high values of honey (until two spoons of tea), this difference between the two curves is very small. 
Table 1. Efficiency of both possible rotation directions.

\begin{tabular}{llll}
\hline & Gear's RPM & Bar's RPM & Efficiency \\
\hline Clockwise & 5174 & 1213 & $23.4 \%$ \\
Counter-clockwise & 4740 & 1083 & $22.8 \%$ \\
\hline
\end{tabular}

Table 2. Gear measured properties.

\begin{tabular}{lllll}
\hline & Tension $(\mathrm{V})$ & Current $(\mathrm{A})$ & Power $(\mathrm{W})$ & Angular frequency $(\mathrm{RPM})$ \\
\hline Gear only & 1.9 & 0.6 & 1.1 & 5174 \\
Coupled magnetic bar & 1.8 & 1.0 & 1.8 & 1213 \\
\hline
\end{tabular}

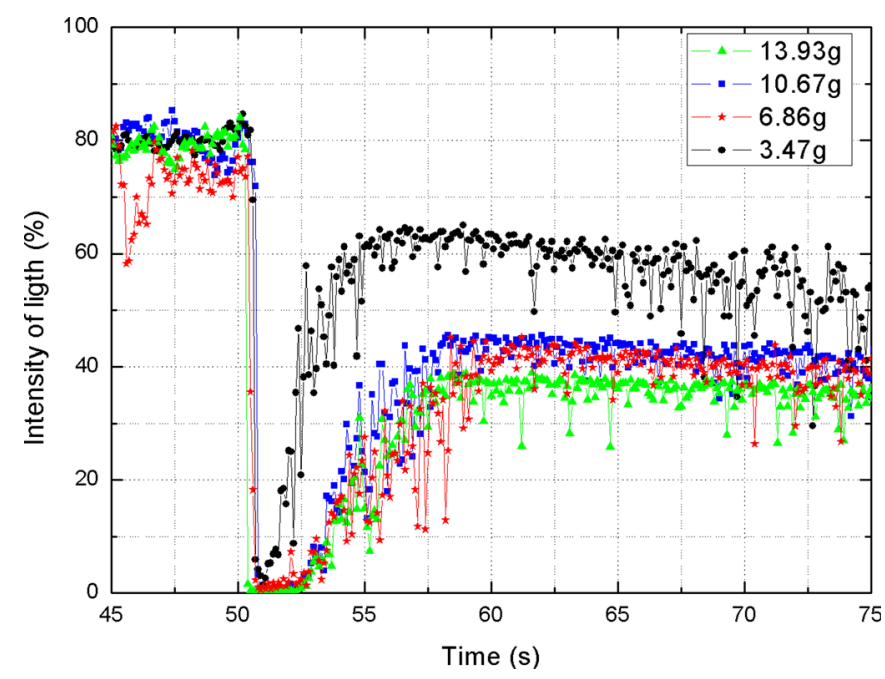

Fig. 8. Luminous intensity (transmittance) test for different honey concentrations at room temperatures of approximately $30.0^{\circ} \mathrm{C}$. Black: half spoon of tea $(2.5 \pm 0.5) \mathrm{mL}$. Red: one spoon $(5.0 \pm 0.5) \mathrm{mL}$. Blue: one and a half spoon $(7.5 \pm 0.5) \mathrm{mL}$. Green: two spoons $(10.0 \pm 0.5) \mathrm{mL}$.

\subsection{Influence of temperature}

Tea can be made at many different temperatures. But to be able to reach an optimal value of time for the stirring process, we must investigate how different temperatures will affect it.

The tests shown in Figure 8 were all made with the addition of $5.0 \mathrm{~mL}$ of honey, the approximate value of a spoon, measured with a syringe $( \pm 0.5 \mathrm{~mL})$. Initial and final temperatures were measured and the average was considered the temperature of the process. Temperature measurements were obtained with a thermometer $\left( \pm 0.5{ }^{\circ} \mathrm{C}\right)$. Results show that temperature has a great influence on the time necessary to reach homogeneity.

From the Table 3 , it is possible to state that temperature and time for mixing successfully (reaching homogeneity) are inversely proportional.

With higher temperatures, the time for homogeneity falls drastically. So, in order to get a faster solubilization time, temperatures above the ambient temperature are needed. Further investigation with temperatures between 32.0 and $50.0^{\circ} \mathrm{C}$ will be done in future experiments.

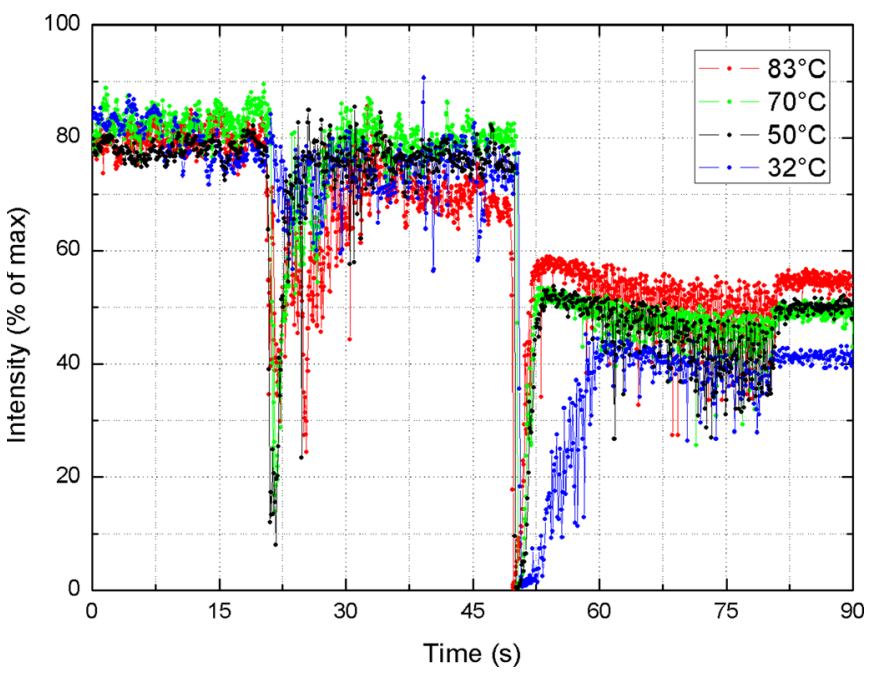

Fig. 9. Luminous intensity (transmittance) test for different temperatures of tea and $(5 \pm 0.5) \mathrm{mL}$ of honey. The graph shows how the temperature is related to the time for reaching homogeneity.

\section{Discussion}

When tea is poured into the vessel and transmittance is started to be measured, it shows a constant value. Then, when honey is poured in the tea's surface, creates fluctuations on transmittance always when honey crosses the laser path. When the stirrer is turned on and creates a vortex, the transport mechanisms are favored and dissolves honey through the tea. When transmittance becomes constant again (now in a lower value than the initial one), we can consider that honey concentration is homogeneous through the mixture. The lower transmittance level indicates the attenuation caused by light absorpted and light scattered by the honey molecules.

Concentration changes the mixing time because a greater quantity of honey molecules in the medium takes longer to separate from each other, once they are kept together by inter-molecular forces and collide with water molecules, according to collision theory. These non-diluted molecules keep scattering laser's light, and transmittance takes a longer time to reach stability. 

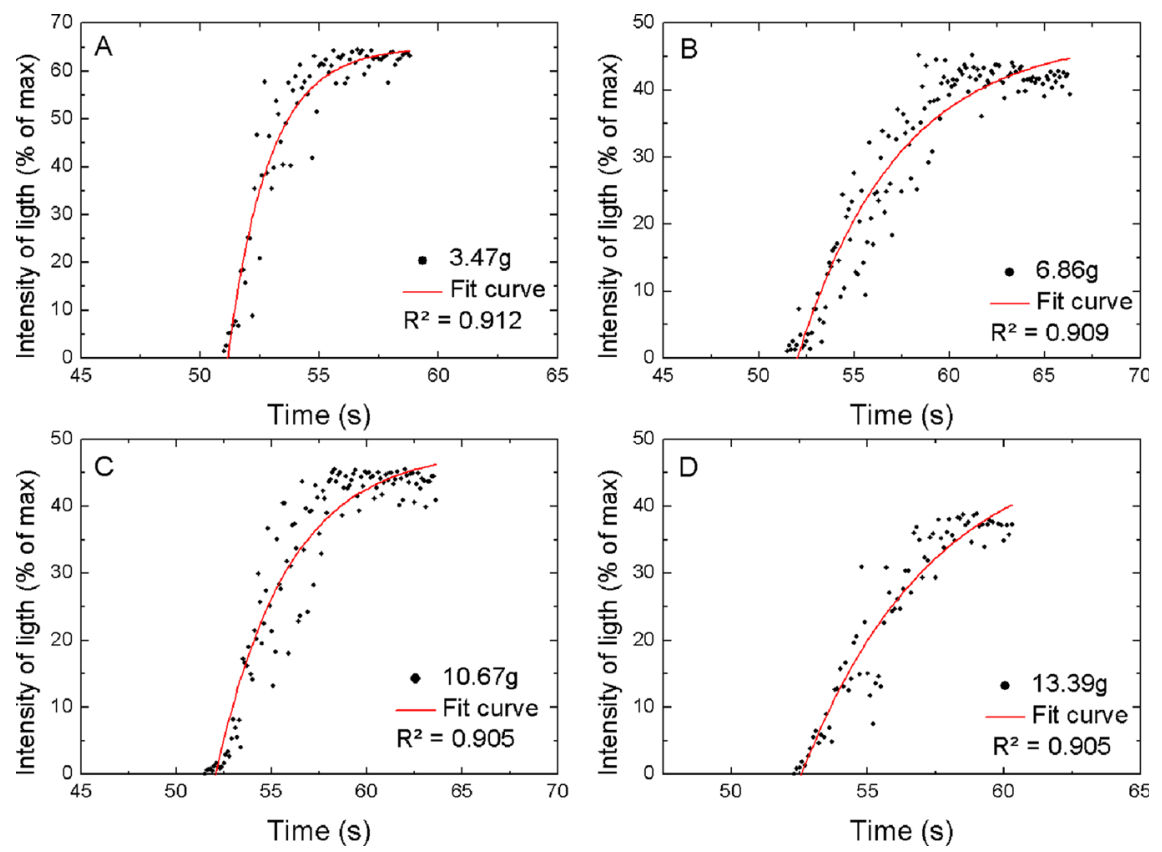

Fig. 10. Fits of transient transmittance curves for different honey concentrations $\left(30^{\circ} \mathrm{C}\right)$.

Table 3. Necessary time $(\tau)$ to reach homogeneity for $5.0 \mathrm{~mL}$ of honey in $250.0 \mathrm{~mL}$ of tea as a function of tea's temperature.

\begin{tabular}{lc}
\hline Temperature $\left({ }^{\circ} \mathrm{C}\right)$ & $(\mathrm{s}) \tau$ \\
\hline 32.0 & $4.4 \pm 0.4$ \\
50.0 & $1.5 \pm 0.2$ \\
70.0 & $1.3 \pm 0.1$ \\
83.0 & $1.4 \pm 0.1$ \\
\hline
\end{tabular}

We can see that lower concentration values of honey are mixed faster. Although, among high values of honey (until two spoons of tea), the difference between the two curves is very small (Figs. 9 and 10). This happens because a higher initial concentration of honey takes more time to be spread homogeneously through the vessel.

It was also possible to observe that the higher the temperature of the tea, the faster the mixture becomes homogeneous (Fig. 8 and Tab. 3). A possible explanation is that hot water has different properties than cold water (such as viscosity, density and surface tension), which offers lower resistance to the mixing process.

Hot tea final transmittance values are higher than colder teas, which means that the molecular aggregates are smaller: The high temperature helps to separate more the molecular aggregates, generating smaller particles. This reduces the effect of Mie's scattering, which scatters more light than the increased effects of Rayleigh scattering and absorption.

We fit the experimental data with equation (3), analogous to equation (2):

$$
I_{a}(t)=I_{F}-B e^{\frac{-t}{\tau}}
$$

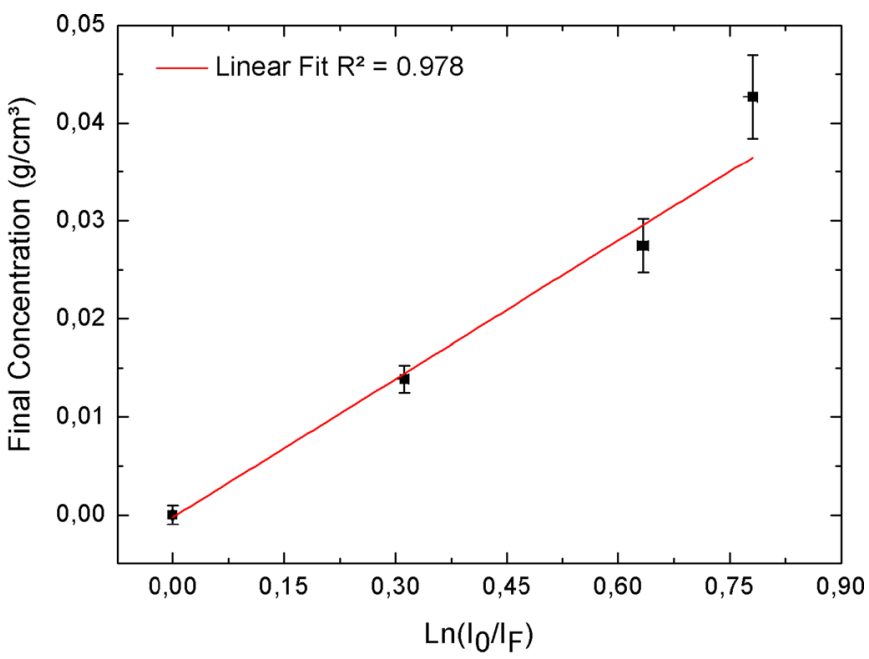

Fig. 11. Linear relation between final concentration and $\ln \left(\frac{I_{0}}{I_{f}}\right)$.

where $I_{a}$ is the measured attenuated light intensity; $I_{f}$ is the final equilibrium light intensity; $B$ is a proportionality constant and $\tau$ is the time constant of the system.

Manipulating equation (3) with equation (1) by equating the attenuated light intensities, yields equation (4), which expresses the medium particle density $N$, as shown in Figure 11:

$$
N(t)=\frac{-1}{\sigma d} \ln \left(\frac{I_{a}(t)}{I_{0}}\right) .
$$

The particles density $N$ can be approximated to concentration using a quotient between Avogadro's number and molar mass. The plot was made at the limit where the transient process had already finished, so it is 

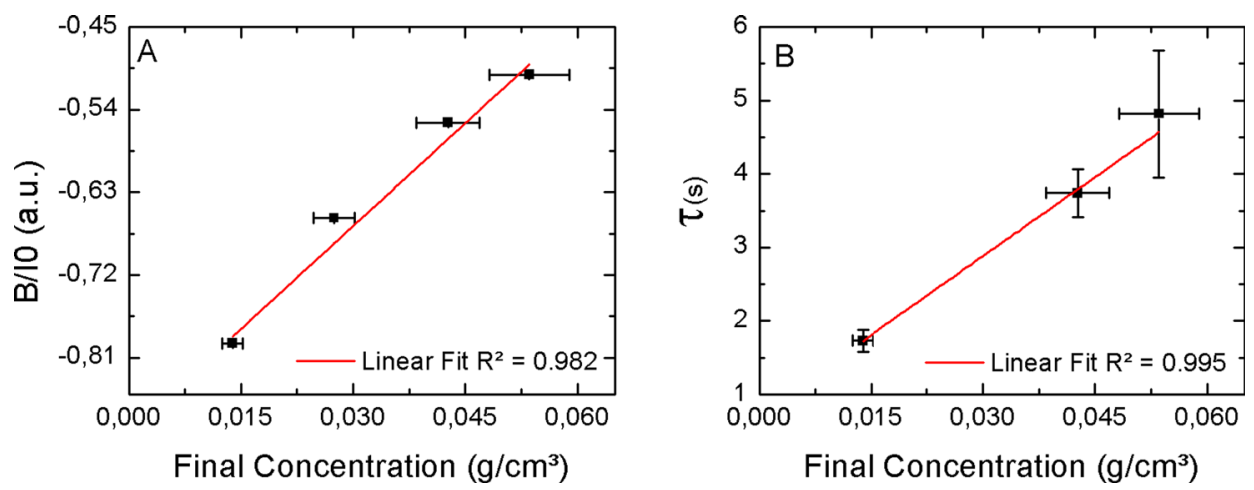

Fig. 12. (A) Linear relation between $\frac{B}{I_{0}}$ and $\varphi_{\text {final }}$; (B) Linear relation between $\tau$ and $\varphi_{\text {final }}$.

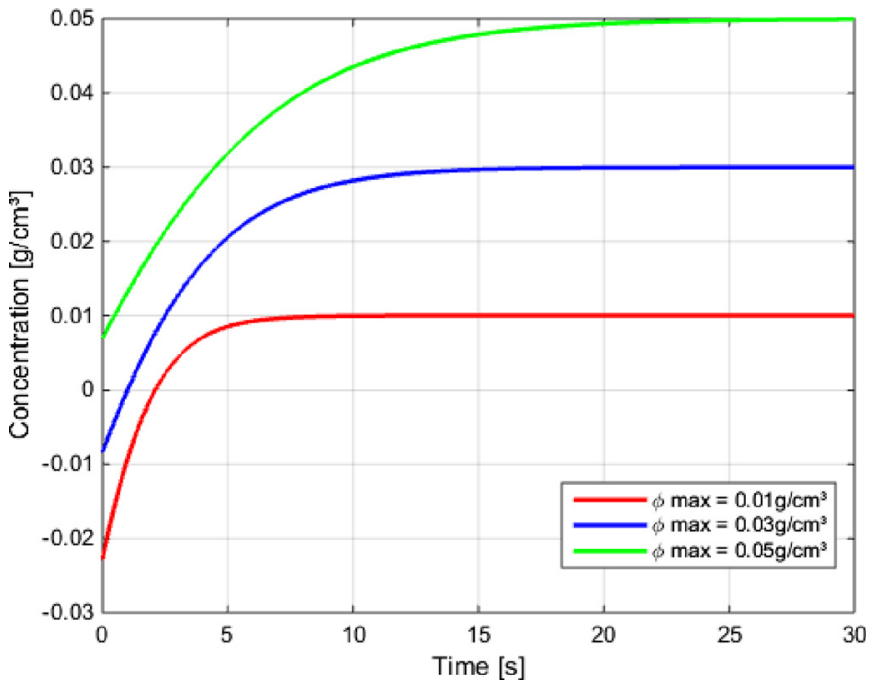

Fig. 13. Concentration in function of time for three different final concentrations $\varphi_{\text {final }}$.

possible to imply that the concentration considered is the (measurable) final concentration and the intensity is the constant intensity at the end of the process.

The validity of the light interaction theoretical model, which provides us with a linear relation between light absorption $A=-\ln \frac{I_{a}}{I_{0}}$ and the cross-section $\sigma$, according to equation (1), can be proved by the experimental data, as shown in Figure 11, which shows a clear linear relation [11]. Such relation allows us then to experimentally estimate the cross section, based on the empirical determination of the other parameters and by measuring the ratio between the attenuated and incident light intensities. The experimental idea is analogous that used for measurements of extinction by the aerosol clouds, as discussed in [7].

The experimental data allows us to determine the angular coefficient of the line in Figure 11. Taking the other parameters' experimental measurement: $d=(3.73 \pm 0.05) \mathrm{cm}$ (optical path, empirically determined); and $m=180.16 \mathrm{~g}$ $\mathrm{mol}^{-1}$ (which is a molar mass representative for Glucose and Fructose); we get: $\sigma=1.7 \times 10^{-25} \mathrm{~m}^{2}=1.7 \mathrm{kbarn}$ as the cross-section. This cross section determines the probability of an incident photon interact with the molecule of honey. Using this value is possible to determine the molar extinction coefficient and compare it with other values in literature of different organic molecules [6].

\section{Dead end}

In this section, we present some other experimental setups which were considered (here only qualitatively described). Afterward, we present some observations about our optical setup and discuss the concentration even further, investigating the correlation between the final concentration of honey and total time for solubilization, which, as will be discussed, did not lead to any conclusion.

\subsection{The experimental setup}

The first task in order to solve the problem was to determine the stirrer's design, that is, what kind of stirrer was going to be built. Of course, though, we had two guidelines directing such choice: the stirrer should be optimized so that the mixing would occur in the minimal time, and it should be powered by a single $1.5 \mathrm{~V}$ battery. In fact, the power limitation was the major caveat, because it actually prevented some designs to even work. For example, we tried to build a mechanical stirrer, like a blender, i.e., such that the rotating motor was placed in direct contact with the tea. The design did not work. We also tried to couple a paddle to the rotating motor, and then directly place the rotating paddle within the tea (like a mixer). In this case, the paddles did rotate within the tea, but no vorticity was observed so that the device was certainly far from optimized for mixing tea and honey.

The magnetic stirrer, on the other hand, worked well from the very beginning, since it produced a vortex from the bottom - where the honey had to be initially located -, which made the stirring process more favorable and faster. It was then clear that this should be the chosen design.

We also tried other methods, besides the light scattering, to determine the homogeneity of the mixture. For example, we tried to use an electrochemical method to measure the difference in electrical resistance of pure tea and the tea with honey. We hoped to track how the difference evolved with time, which would allow an approach similar to that described for the light scattering. However, the experimental results showed no relevant information in this case due to the lack of sensibility of the devices used in these measurements. 

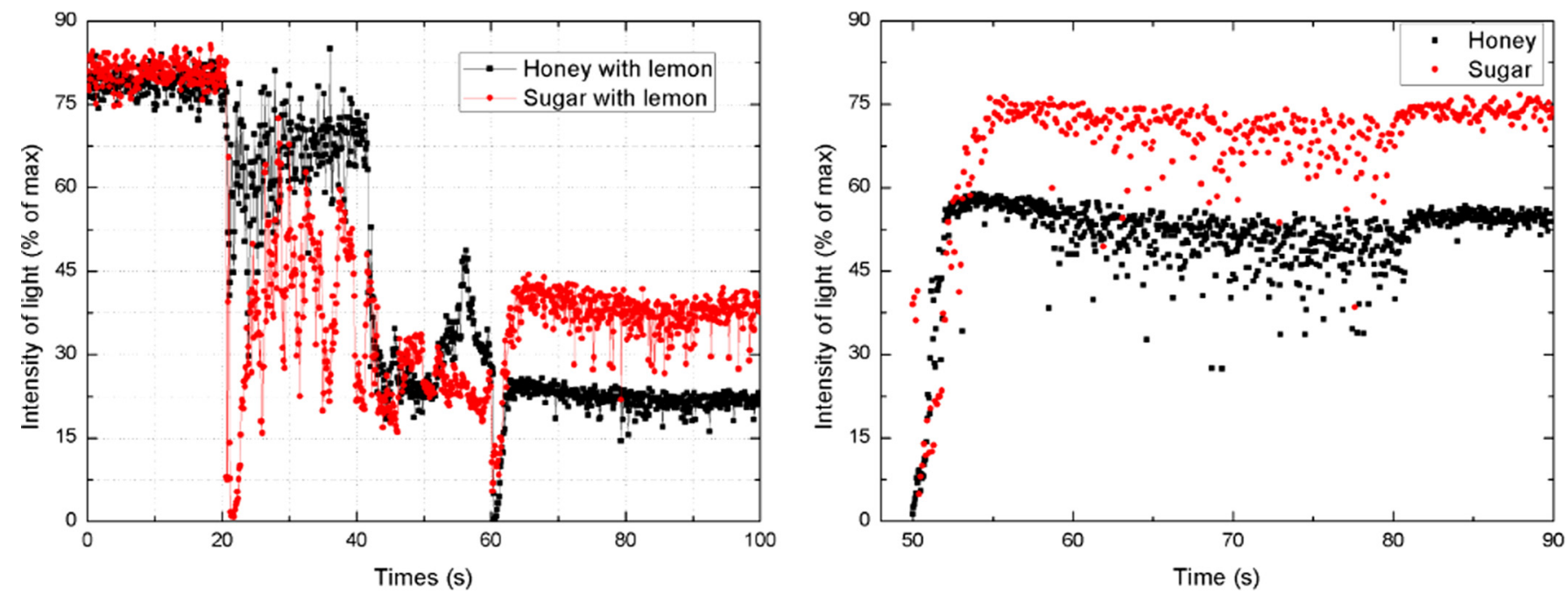

Fig. 14. Left: Fits of stability curves for different honey concentrations. Comparison between the addition of lemon in two cases: tea with $5.0 \mathrm{~g}$ of sugar and tea with $5.0 \mathrm{~mL}$ of honey. The temperature was maintained constant in $T=34.0^{\circ} \mathrm{C}$. Right: comparison between honey (measurement of $5.0 \pm 0.5 \mathrm{~mL}$ ) and sugar (measurement of $5.0 \pm 0.1 \mathrm{~g}$ ) when added to tea at $(83.0 \pm 0.5)^{\circ} \mathrm{C}$.

\subsection{Further investigation on concentration}

We made a try to obtain an expression for concentration in function of time to demonstrate the effects of the experimental apparatus used, but as the following results show, more experiments are needed to guarantee the validity of the functions for each variable of interest (temperature, relaxation time, solute volumes and initial solvents, for example). Some of the results of these approximations were consistent, however, the lack of new experiments does not guarantee valid points in this case.

Using the light attenuation equation, theoretically, it could be possible to find a relation between the final concentration of honey and the total time for solubilization, and the final concentration of honey, as the following construction shows.

The medium particle density can be calculated in terms of the solution concentration, as $N=\rho \frac{N_{a}}{m}$, where $\rho$ is the particle's density; $N_{A}$ is Avogadro's constant and $m$ is its molar mass, which yields equation (5), for the concentration $\varphi$ as a function of time.

$$
\varphi(t)=\frac{-m}{\sigma d N_{A}} \ln \left(\frac{I_{f}}{I_{0}}-\frac{B}{I_{0}} e^{\frac{-t}{\tau}}\right) .
$$

For long periods of time, the transient term may be neglected (by taking $t \rightarrow \infty$ ), which yields in a final concentration $\varphi_{\text {final }}$ given by equation (6).

$$
\phi_{\text {final }}=\frac{-m}{\sigma d N_{A}} \ln \left(\frac{I_{f}}{I_{0}}\right) .
$$

On the other hand, we're interested in the mathematical model for $\varphi(t)$. To get that, we must determine equation (5)'s parameters, which is made by the fitting of the experimental data. Figure $12 \mathrm{~A}$ and B shows the relation between $\frac{B}{I_{0}}$ and $\varphi_{\text {final }}$ and $\tau$ and $\varphi_{\text {final }}$ respectively, which are both linear.
With that, the model was plotted with a MATLAB script (Fig. 13). The negative values for $\varphi$ happen due to the displacement of the adjustment time, as an approximation which guarantees $\varphi>0$ must take into account the initial time of measurement $t_{0}$ in equation (3).

The fit equation found was as described in equation (7),

$$
\varphi(t)=-a \ln \left(b-c e^{\frac{-t}{\tau^{\prime}}}\right),
$$

where $b\left(\varphi_{\text {final }}\right)=e^{\frac{-\varphi_{\text {final }}}{a}}, c\left(\varphi_{\text {final }}\right)=7.462 \varphi_{\text {final }}-0.891$, and $\tau^{\prime}\left(\varphi_{\text {final }}\right)=71.546 \varphi_{\text {final }}+0.737$.

As Figure 12B shows, the experimental results when obtained and compared to these approximations show a linear relation with a large error bar that leads to a dead end in this case. Although we see a good correlation of the $B$ parameter in the equation with the final concentration, it is not possible to affirm that a linear relation between the time for solubilization and the final concentration exists. However, more experimental repetitions are encouraged to prove or disprove this approximation.

\section{Conclusion}

The proposed method to mix tea and honey consists to use a magnetic stirrer, which generates a vortex in the solvent fluid. This vortex is responsible to mechanically spread the honey aggregates through the fluid. Considerations about optimizing the device are discussed, respecting the criteria of continuous mixing and with a single $1.5 \mathrm{~V}$ battery.

The measurement of transmittance of the light from a laser has been chosen as the indication that homogeneity was reached. Discussing the theory among this process, it was shown that the results are consistent to other literatures, also determining the light extinction cross section.

It has been demonstrated that transient light attenuation can be used to measure the necessary time of mixing. The effects of variating temperature and honey concentration were shown, demonstrating that a lower concentration of honey in a hotter tea has the shortest necessary mixing time. 
Extra results on the Appendix show more investigations when considering the stirrer dynamics and the tea with sugar and lemon. Tea with honey or sugar presents a similar response.

We would like to thank Prof. Dr. Antonio Alvaro Ranha Neves for helping us on every stage of experimental procedures and writing of the first versions of this paper.

\section{A Appendices}

\section{A.1 Rotational movement of the stirrer bar}

The magnets produce a magnetic field which is approximately horizontal at the height of the magnetic stirrer bar, as shown in Figure 2 (lateral view) of main text. As the magnet rotates, a magnetic torque is induced to the bar, thanks to their magnetic coupling. The torque $\tau_{B}$ (where we use boldface symbols to indicate vector quantities) is given by equation (8):

$$
\boldsymbol{\tau}_{\boldsymbol{B}}=-\mu B_{0} \sin (\theta-\omega t) z,
$$

where $\mu$ is the magnetic dipole moment of the magnetic stirrer bar; $B_{0}$ is the magnitude of the magnetic field from the magnets at the location of the height of the bar; $\theta$ is the orientation of the stirrer bar with relation to the axis shown in Figure 2 (upper view); $\omega$ is the angular frequency of the rotating magnets attached to the axis of a DC motor and $t$ is the time in seconds counting from the moment in which the motor is turned on. The argument $\theta-\omega t$ can be found analyzing an upper view of the setup, as shown in Figure 2 (upper view). As long as the magnets are close enough to the bar, the magnetic coupling between them is strong enough to produce small angular displacements between them, so that we can promptly approximate: $\sin (\theta-\omega t) \approx \theta-\omega t$.

Both tea and honey offer a mechanical resistance to the rotation of the bar, which is then expressed by the drag torque $\boldsymbol{\tau}_{\boldsymbol{D}}$ given by: $\boldsymbol{\tau}_{\boldsymbol{D}}=-k \dot{\theta} \mathbf{z}$, where $k$ is the drag coefficient related to the fluid's viscosity and the bar's geometry and $\dot{\theta}$ is the magnetic bar's angular velocity. The net mechanical torque, in terms the of the bar's angular acceleration $\theta$ and its moment of inertia $I$, is given by:

$$
\boldsymbol{\tau}_{\boldsymbol{R}}=\boldsymbol{\tau}_{\boldsymbol{B}}+\boldsymbol{\tau}_{\boldsymbol{D}}=I \theta \hat{z} .
$$

The resulting equation of motion for the magnetic stirrer bar, considering all torques acting in the system in the $\mathbf{z}$ direction, is presented in equation (10),

$$
I \theta+k \dot{\theta}+\mu B_{0} \theta=\mu B_{0} \omega t
$$

The solution of differential equation shown in equation (10) is:

$$
\begin{aligned}
\theta(t) & =c_{1} \exp \left(-\frac{t}{2 I}\left(\sqrt{k^{2}-4 I B_{0} \mu}+k\right)\right) \\
& +c_{2} \exp \left(\frac{t}{2 I}\left(\sqrt{k^{2}-4 I B_{0} \mu}-k\right)\right)+t \omega \\
& -\frac{k \omega}{B_{0} \mu}
\end{aligned}
$$

where $c_{1}$ and $c_{2}$ are two arbitrary integration constants fixed by the initial conditions $\theta(t=0)=\dot{\theta}(t=0)=0$; and $k$ is the drag coefficient. The first two terms in equation (11) represent the transient behavior of the solution, since they go to zero as the time increases. In fact, that is easily seen by noticing that the first exponent is negative and that the second exponent is such that $k^{2}-4 I B_{0} \mu \leq k^{2}$. Therefore, as time passes, the transient regime is surpassed and the magnetic bar's angle increases linearly with time.

Thus, the magnetic bar will follow a circular motion, driven by the magnetic torque transferred by the rotating magnets and damped by the drag torque, until the steady state is achieved, from which point the bar will keep its angular velocity constant.

\section{A.2 Adding lemon to the tea}

Adding, literally, a slice of lemon, brought many problems to our setup considering the mechanical interference with the bar's movement in the magnetic stirrer. However, to study its influence in a mix with honey and sugar, a slice of lemon was squeezed and the quantity of liquid contained in it (lemon juice) was measured with a syringe $(2.0 \pm 0.5 \mathrm{~mL})$. In Figure 14 (left), the plateau of homogeneity is notably different due to the optical effects presented in the subsection G. When $t=20 \mathrm{~s}$, there is a notable difference between both patterns. This happens given the inter-molecular interaction between the acids present in lemon and the sugar complexes which mainly constitute the honey, which increases the solubility by breaking the sugar complexes into smaller polymers.

\section{A.3 Comparing sugar and honey}

When sugar is added instead of honey, the time necessary to reach homogeneity (at same temperature) is almost equal, but sugar's intensity stabilization curve is higher because after homogeneity, the tea with sugar solution medium is optically closer to the medium with just tea (due to factors such as - though not only - sugar's smaller size), than the honey and tea solution medium which, after homogeneity, has optical properties more expressively different from those with just tea. The difference in the stabilization intensity then shows itself as a clear way to indirectly determine which one was mixed with the tea. In Figure 14 (right) we see the plateau differences due to inter-molecular effects.

\section{References}

1. L.T. Fan, J.T. Tseng, Apparent diffusivity in honey-water system, J. Food Sci. 32, 633-636 (1967), DOI:10.1111/ j.1365-2621.1967.tb00852.x

2. G. Halasz, B. Gyre, I.M. Jnosi, K.G. Szab, T. TL, Vortex flow generated by a magnetic stirrer, Am. J. Phys. 75, 1092-1098 (2007)

3. J.W. White, L.W. Doner, Honey composition and properties, Beekeeping in the United States Agriculture Handbook, Philadelphia, Vol. 335, (1980), pp. 82-91 
4. D.J. Lockwood, Rayleigh and Mie scattering, in Encyclopedia of Color Science and Technology, Springer, New York, NY, 2016, pp. 1-12, Print ISBN 978-1-4419-8070-0, Online ISBN 978-1-4419-8071-7

5. C. Bohren, D. Huffman, Absorption and Scattering of Light by Small Particles (Wiley-Interscience, Canada, 1998)

6. K. Singh, G.K. Sandhu, B.S. Lark, S.P. Sud, Molar extinction coefficients of some carbohydrates in aqueous solutions, Pramana J. Phys. 58 (2002)

7. S.K. Friedlander, Smoke, Dust, and Haze: Fundamentals of Aerosol Dynamics (Oxford University Press, 2000), ISBN 10: 0195129997

8. M.O. Mcanally, et al., Quantitative determination of the differential raman scattering cross sections of glucose by femtosecond stimulated raman scattering, Anal. Chem. 89, 6931-6935 (2017), DOI:10.1021/acs.analchem.7b01335
9. S. derholm, H.Y. Roos, N. Meinander, M. Hotokkka, Raman spectra of fructose and glucose in the amorphous and crystalline states, J. Raman Specrtosc. 30, 1009-1018 (1999), <https://doi.org/10.1002/(SICI)1097-4555(199911) 30:11<1009::AID-JRS436>3.0.CO;2-\#>

10. A.T. Young, Rayleigh scattering, Phys. Today 35, 42-48 (1982)

11. G. Mie, Beitrge zur Optik trber Medien, speziell kolloidaler Metallsungen, Ann. Phys. 330, 377-445 (1908)

12. T. Rosenrn, G. Kiss, M. Bilde, Cloud droplet activation of saccharides and levoglucosan particles, Atmos. Environ. 40, 1794-1802 (2006), https://doi.org/10.1016/j.atmos env.2005.11.024, ISSN 1352-2310

13. E. Collett, Field guide to polarization (SPIE Press, Bellingham, 2005), Vol. 15

Cite this article as: Henrique Ferreira, Ricardo R. Gitti, Andre J. Ferreira-Martins, Matheus Pessoa, Pedro R. Sardelich, Isabela S. Bijotti , Method to mix tea with honey and use of transient light attenuation as an indicator of homogeneity, Emergent Scientist 2, 2 (2018) 